\title{
Uncertainty principle and superradiance
}

\author{
Wen-Xiang Chen* \\ Department of Astronomy, \\ School of Physics and Materials Science, \\ GuangZhou University
}

\begin{abstract}
Associate Professor Hasegawa Yuji of the Vienna University of Technology and Professor Masaaki Ozawa of Nagoya University and other scholars published empirical results against Heisenberg's uncertainty principle on January 15, 2012.They got a measurement result with a smaller error than the Heisenberg uncertainty principle, which proved the measurement advocated by the Heisenberg uncertainty principle. This article follows the method I used to study superradiation and connects the uncertainty principle with the superradiation effect. I found that under the superradiation effect, the measurement limit of the uncertainty principle can be smaller.
\end{abstract}

Keywords: Uncertainty principle, superradiance, Wronskian determinant

\section{INTRODUCTION}

In quantum mechanics, the uncertainty principle states that the position and momentum of a particle cannot be determined at the same time. The smaller the position uncertainty, the greater the momentum uncertainty, and vice versa. The same is true. For different cases, the connotation of uncertainty is different. It can be the observer's lack of a certain amount of information, it can also be a certain amount of measurement error, or an ensemble of similar preparations. The statistical diffusion value of the system. Werner Heisenberg published the paper "On the Physical Connotation of Quantum Theory Kinematics and Mechanics" in 1927, giving the original heuristic exposition of this principle, hoping to successfully qualitatively analyze and express the physical properties of simple quantum experiments. This principle is also known as the Heisenberg uncertainty principle. Later in the same year, Earl Kennard rigorously formulated mathematically the uncertainty relationship between position and momentum. Two years later, Howard Robertson promoted Kennard's relationship.

Similar uncertainty relations also exist between energy and time, angular momentum and angle and other physical quantities. Since the uncertainty principle is the basic theory of quantum mechanics, many general experiments often involve some questions about it. Some experiments will specifically test this principle or similar principles. For example, testing the digital-phase uncertainty principle that occurs in superconducting systems or quantum optical systems. The related research on the uncertainty principle can be used to develop the low-noise technology required by the gravity wave interferometer.

Superradiance-The method originally called Klein's Paradox can be traced back to Klein's original paper, who first studied the Dirac equation in the presence of a step potential. He showed that the electron beam propagating in a region where the barrier $\mathrm{V}$ is large enough may appear without the exponential damping expected by the non-

\footnotetext{
*Electronic address: wxchen4277@qq.com
} 
relativistic quantum tunneling process. When trying to understand whether this result is an artifact of the step potential used by Klein, Sauter found that the nature of the process has nothing to do with the details of the barrier, although the probability of propagation decreases with decreasing slope. This phenomenon was first called "Klein's Paradox" by Sauter in 1931.

Further research conducted by Hong De in 1941 now deals with charged scalar fields and the Klein-Gordon equation, and the results show that when the potential is strong enough, the step potential produces a pair of charged particles. Hunde tried-but failed-to get the same result for fermions. It is worth noting that this result can be regarded as a precursor to the results of Schwinger and Hawking's modern quantum field theory. The latter shows that spontaneous pairs are possible in the presence of strong electromagnetic fields and gravitational fields of bosons and fermions. . In fact, we already know today that the resolution of the "old" Klein paradox is due to the formation of particleantiparticle pairs at the barrier, which explains the unattenuated transmission part.

In 1972, Press and Teukolsky[15] proposed that It is possible to add a mirror to the outside of a black hole to make a black hole bomb (according to the current explanation, this is a scattering process involving classical mechanics and quantum mechanics[1, 3, 9, 10, 13, 14, 16]).

When a bosonic wave is impinging upon a rotating black hole, the wave reflected by the event horizon will be amplified if the wave frequency $\omega$ lies in the following superradiant regime[11, 12, 15, 17, 18]

$$
0<\omega<m \Omega_{H}, \Omega_{H}=\frac{a}{r_{+}^{2}+a^{2}}
$$

where $m$ is azimuthal number of the bosonic wave mode, $\Omega_{H}$ is the angular velocity of black hole horizon. This amplification is superradiant scattering. Therefore, through the superradiation process, the rotational energy of the black hole can be extracted. If there is a mirror between the black hole's horizon and infinite space, the amplified wave will scatter back and forth and grow exponentially, which will cause the black hole's superradiation to become unstable.

Associate Professor Hasegawa Yuji of the Vienna University of Technology and Professor Masaaki Ozawa of Nagoya University and other scholars published empirical results against Heisenberg's uncertainty principle on January 15, 2012[8]. They used two instruments to measure the spin angle of neutrons and calculated them, and they got a measurement result with a smaller error than the Heisenberg uncertainty principle, which proved the measurement advocated by the Heisenberg uncertainty principle. The limit is wrong. However, the uncertainty principle is still correct, because this is the inherent quantum nature of the particle.

This article follows the method I used to study superradiation and connects the uncertainty principle with the superradiation effect. I found that under the superradiation effect, the measurement limit of the uncertainty principle can be smaller.

\section{THE SUPERRADIATION EFFECT OF BOSON SCATTERING}

We get the Klein-Gordon equation

$$
\Phi_{; \mu}{ }^{; \mu}=0,
$$


where we defined $\Phi_{; \mu} \equiv\left(\partial_{\mu}-i e A_{\mu}\right) \Phi$ and $e$ is the charge of the scalar field.We get $A^{\mu}=\left\{A_{0}(x), 0\right\}$, and $e A_{0}(x)$ can be equal to $\mu$ (where $\mu$ is the mass).

$$
A_{0} \rightarrow\left\{\begin{array}{ll}
0 & \text { as } x \rightarrow-\infty \\
V & \text { as } x \rightarrow+\infty
\end{array}\right. \text {. }
$$

With $\Phi=e^{-i \omega t} f(x)$, which is determined by the ordinary differential equation

$$
\frac{d^{2} f}{d x^{2}}+\left(\omega-e A_{0}\right)^{2} f=0
$$

We see that particles coming from $-\infty$ and scattering off the potential with reflection and transmission amplitudes $\mathcal{R}$ and $\mathcal{T}$ respectively. With these boundary conditions, the solution to behaves asymptotically as

$$
\begin{gathered}
f_{\text {in }}(x)=\mathcal{I} e^{i \omega x}+\mathcal{R} e^{-i \omega x}, x \rightarrow-\infty, \\
f_{\text {in }}(x)=\mathcal{T} e^{i k x}, x \rightarrow+\infty
\end{gathered}
$$

where $k= \pm(\omega-e V)$.

To define the sign of $\omega$ and $k$ we must look at the wave's group velocity. We require $\partial \omega / \partial k>0$, so that they travel from the left to the right in the $x$-direction and we take $\omega>0$.

The reflection coefficient and transmission coefficient depend on the specific shape of the potential $A_{0}$. However one can easily show that the Wronskian

$$
W=\tilde{f}_{1} \frac{d \tilde{f}_{2}}{d x}-\tilde{f}_{2} \frac{d \tilde{f}_{1}}{d x},
$$

between two independent solutions, $\tilde{f}_{1}$ and $\tilde{f}_{2}$, of is conserved. From the equation on the other hand, if $f$ is a solution then its complex conjugate $f^{*}$ is another linearly independent solution. We find $|\mathcal{R}|^{2}=|\mathcal{I}|^{2}-\frac{\omega-e V}{\omega}|\mathcal{T}|^{2}$. Thus, for $0<\omega<e V$, it is possible to have superradiant amplification of the reflected current, i.e, $|\mathcal{R}|>|\mathcal{I}|$. There are other potentials that can be completely resolved, which can also show superradiation explicitly.

\section{THAT RESULT CONTAINED SOME CONCLUSIONS THAT VIOLATED UNCERTAINTY PRINCIPLE}

The principle of joint uncertainty shows that it is impossible to make joint measurement of position and momentum, that is, to measure position and momentum simultaneously, only approximate joint measurement can be made, and the error follows the inequality $\Delta x \Delta p \geq 1 / 2$ (in natural unit system).We find $|\mathcal{R}|^{2}=|\mathcal{I}|^{2}-\frac{\omega-e V}{\omega}|\mathcal{T}|^{2}$, and we know that $|\mathcal{R}|^{2} \geq-\frac{\omega-e V}{\omega}|\mathcal{T}|^{2}$ is a necessary condition for the inequality $\Delta x \Delta p \geq 1 / 2$ to be established[2, 8]. We can preset the boundary conditions $e A_{0}(x)=y \omega$ (which can be $\mu=y \omega$ )[4][5][6][7], and we see that when $y$ is relatively large(according to the properties of the boson, $y$ can be very large), $|\mathcal{R}|^{2} \geq-\frac{\omega-e V}{\omega}|\mathcal{T}|^{2}$ may not hold.In the end,we can get $\Delta x \Delta p \geq 1 / 2$ may not hold. 


\section{SUMMARY}

This article follows the method I used to study superradiation and connects the uncertainty principle with the superradiation effect. I found that under the superradiation effect, the measurement limit of the uncertainty principle can be smaller.

[1] Berti, E., Cardoso, V. and Casals, M. [2006], 'Eigenvalues and eigenfunctions of spin-weighted spheroidal harmonics in four and higher dimensions', Physical Review D 73(2), 024013.

[2] Brito, R., Cardoso, V. and Pani, P. [2015], 'Superradiance', Lect. Notes Phys 906(1), 1501-06570.

[3] Cardoso, V., Dias, O. J., Lemos, J. P. and Yoshida, S. [2004], 'Black-hole bomb and superradiant instabilities', Physical Review D 70(4), 044039.

[4] Chen, W.-X. [2020a], 'Chronology protection conjecture may not hold', SSRN Electronic Journal .

URL: https://dx.doi.org/10.2139/ssrn.3645919

[5] Chen, W.-X. [2020b], 'The possibility of the no-hair theorem being violated', Available at SSRN 3569639.

URL: https://dx.doi.org/10.2139/ssrn.3569639

[6] Chen, W.-X. [2020c], 'The strong cosmic censorship conjecture may be violated', SSRN Electronic Journal .

URL: https://dx.doi.org/10.2139/ssrn.3610419

[7] Chen, W.-X. and Huang, Z.-Y. [2019], 'Superradiant stability of the kerr black hole', International Journal of Modern Physics D.

URL: https://doi.org/10.1142/S0218271820500091

[8] Erhart, Jacqueline; stephan Sponar, G. S. G. B. M. O. Y. H. [2012], 'Experimental demonstration of a universally valid error-disturbance uncertainty relation in spin-measurements', Nature Physics .

[9] Greiner, W., Müller, B. and Rafelski, J. [1985], Evolution of the vacuum state in supercritical potentials, in 'Quantum Electrodynamics of Strong Fields', Springer, pp. 257-299.

[10] Hartman, T., Song, W. and Strominger, A. [2010], 'Holographic derivation of kerr-newman scattering amplitudes for general charge and spin', Journal of High Energy Physics 2010(3), 118.

[11] Herdeiro, C. A. and Radu, E. [2014], 'Kerr black holes with scalar hair', Physical review letters 112(22), 221101.

[12] Kerr, R. P. [1963], 'Gravitational field of a spinning mass as an example of algebraically special metrics', Physical review letters 11(5), 237.

[13] Manogue, C. A. [1988], 'The klein paradox and superradiance', Annals of Physics 181(2), 261-283.

[14] Penrose, R. [2002], 'Gravitational collapse: The role of general relativity (reprinted from rivista del nuovo cimento, numero speziale, i, vol 257, 1969)', General Relativity and Gravitation 34(7), 1141-1165.

[15] Press, W. H. and Teukolsky, S. A. [1973], 'Perturbations of a rotating black hole. ii. dynamical stability of the kerr metric', The Astrophysical Journal 185, 649-674.

[16] Teukolsky, S. A. [1973], 'Perturbations of a rotating black hole. 1. fundamental equations for gravitational electromagnetic and neutrino field perturbations', Astrophys. J. 185, 635-647.

[17] Vilenkin, A. [1978], 'Exponential amplification of waves in the gravitational field of ultrarelativistic rotating body', Physics Letters B 78(2-3), 301-303.

[18] Zel'dovich, Y. B. [1972], 'Pis' ma zh. eksp. teor. fiz. 14, 270 (1971)[jetp lett. 14, 180 (1971)]', Zh. Eksp. Teor. Fiz 62 , 2076. 